\title{
Neural-Fuzzy Approach for Power Load Forecasting Analysis
}

\author{
J. Kumaran Kumar \\ Research scholar in SCSVMV \\ university \\ Assistant professor \\ Department of Computer \\ Science and Engineering, \\ Pondicherry Engineering \\ College, \\ Puducherry, India
}

\author{
G. Ravi, PhD. \\ Associate professor \\ Department of Electrical and \\ Electronics Engineering, \\ Pondicherry Engineering \\ College, \\ Puducherry, India
}

\author{
R. Rajkumar \\ PG student \\ Department of Computer \\ Science and Engineering, \\ Pondicherry Engineering \\ College, \\ Puducherry, India
}

\begin{abstract}
This paper presents Neuro-Fuzzy approach for forecasting analysis in power load. Forecasting the power load is a difficult task for a country and both positive and negative load forecasting makes a big problem for the country. An approach that Neuro-Fuzzy model is proposed for forecast power load in this paper. The proposed model a fuzzy back propagation network is constructed and then a fuzzy intersection is applied and after that de-fuzzify the result to generate a crisp value by using Radial Basis Function network (RBF). The proposed model improves the accuracy of power load forecasting. The forecasted results obtained by neuro-fuzzy method were compared with the Artificial Neural Network by using Mean Absolute Percentage Error (MAPE) to measure accuracy of the result. The experimental result shows that the neuro-fuzzy implementations have more accuracy.
\end{abstract}

\section{Keywords}

Artificial Neural Network, Load forecasting, Neuro-fuzzy model, Radial basis function network.

\section{INTRODUCTION}

Power load forecasting is the prediction of future load consumption by using the historical data. The most important sources for social and economic development of all nations are electrical energy. The growth in energy is closely related to growth in economy. Accurate power load forecasting is very critical task for power systems although it is very essential to estimate future power consumption because it reduces production cost and developing the reliability of power systems.

\subsection{Types of load forecasting}

- Short term load forecasting (STLF) - forecasting the power consumption upto 1 day.

- Medium term load forecasting (MTLF) - forecasting the power consumption one day to 1 year.

- Long term load forecasting (LTLF) - forecasting the power consumption more than 1 year.

There are many techniques to solve the problem of forecasting. Those techniques are depending on the types of application, short, medium and long term forecasting.

\section{RELATED WORKS}

This section describes:

- Structure of ANN

- Fuzzy logic

- Multiple linear regression

\subsection{Artificial Neural Network (ANN)}

An ANN [1] is a model that is encouraged by the biological nervous system. The neural network that perform a tasks which is similar to human brain.

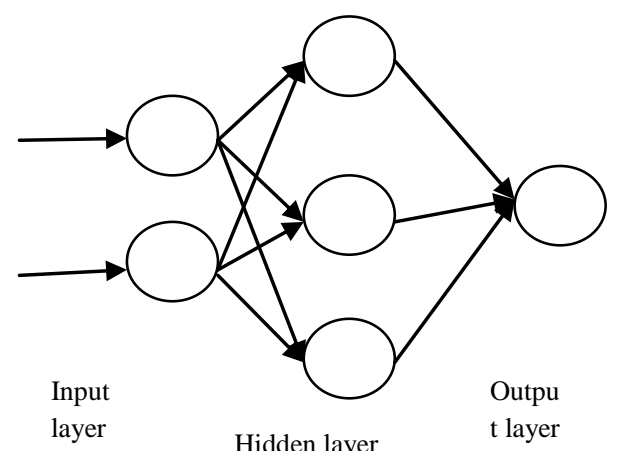

Figure. 1. Structure of ANN model

Multilayer perceptions [2],[3],[4] with back propagation technique [5] are suitable for load forecasting because it helps in the noisy or incomplete in the input data. The structure of ANN model shown in Figure. 1.

The multilayer perceptions (MLP) consist of:
1. Input layer
2. Output layer
3. 1 or more hidden layer.

It is very important to select a correct number of hidden layers and a correct number of neurons in the hidden layer and the connection between them.

\subsection{Fuzzy Logic}

In traditional logic have two valued logic whether true or false. But in fuzzy logic [6], [7] has many valued logic. The value ranges between 0 and 1 . The block diagram of a fuzzy system as shown in Fig. 2. 


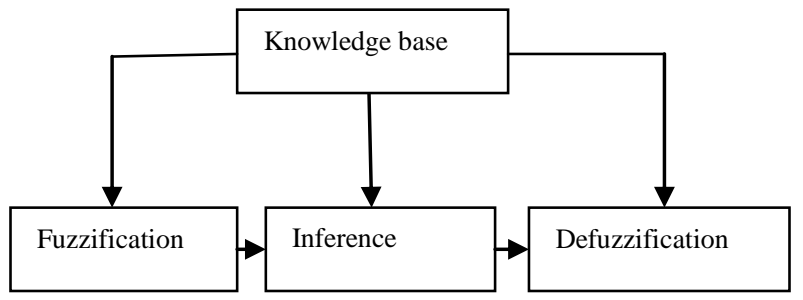

Figure 2. Block diagram of a fuzzy system

\section{Fuzzification}

Fuzzification is the process of scales and map input data to fuzzy sets. It is important to select the shape of the membership function. Usually triangle or trapezoidal membership functions are used. Based on the membership function, the membership values are calculated. Usually the values like low, medium and high.

\section{Fuzzy inference}

It is the method of approximate reasoning. Based on the membership values the IF THEN rules are formed.

\section{Defuzzification}

It is the method of converting the fuzzy output to the forecasted values.

\section{Steps in fuzzy logic model}

1. Proper selection of input and output variables.

2. The input and output variable to be normalized within the range 0 to 1 .

3. The shape of the membership functions for each input and output variables to be selected.

4. Based on the membership function, find the membership value for each input and output.

5. Construct the fuzzy rule for each input and output data, which is also called as training data.

6. By using the training data, calculate the forecasted value.

\subsection{Multi linear regression}

Multi linear regression [8] is a model, the relationship between more than one explanatory variable like temperature, population, GDP etc and dependent variable power consumption. it is the form of regression analysis which is modeled by a least square function. This method is used in most of the practical application, because it easily fit linearly on their unidentified parameters than models which are nonlinearly related to their parameters.

This method is expressed in the form as:

Where,

$$
y(t)=a_{0}+a_{1} x_{1}(t)+\cdots+a_{n} x_{n}(t)
$$

$$
\begin{aligned}
& y(t) \text { is the power consumption } \\
& x_{1}(t), \ldots . x_{n}(t) \text { is the input variables } \\
& a_{\mathbb{0}}, a_{1}, \ldots a_{n \mathbb{1}} \text { is regression coefficients. }
\end{aligned}
$$

The selection of explanatory variables is very important and it is identified on the basis of correlation technique. The regression co-efficient are identified by the least square estimation technique.

\section{ECONOMY FACTOR}

The proper selection of input parameter for load forecasting is very important. There is lot of factors which affects the power consumption. Some of them are as follows:

1. Population.

2. Gross domestic product.

3. Number of households.

4. Amount of $\mathrm{CO}_{2}$ pollution.

5. Oil prices.

6. Electricity price.

7. Temperature.

From the above factors population [13] and GDP [14] are the important factors in power consumption [15]. This is because, if the population and GDP increase, then the power consumption also increases.

The growth in population and GDP will bring a huge increase in power consumption and it is directly proportional to it.

\section{EXISTING MODEL}

An ANN as a system consists of a number of interconnecting elements to solve some specific task. These interconnecting elements in an ANN are known as neurons [9], [10]. Each neuron may have several inputs and only one output. The output of the input layer is send to the hidden layer, which in turn sent to the output layer.The multilayer perceptrons with back propagation learning algorithm [5] is used in ANN model because it is suitable model for long term forecasting, which helps in the non linearity incomplete or noisy in the input data. The network can be trained by adjusting the weight between the neurons. Therefore, the neural network is accepted, based on a similarity of the target and the output. Back-propagation is a regular method of training multilayer artificial network. The inputs are passed to the network, then the output is calculated based on the network selected and the output is compared with the targets. If the output obtained output is not closer to the target value then the learning rule is used to adjust the weights between the neurons in order to get network outputs closer to the targets. Fig. 3 shows the mathematical model of neurons.

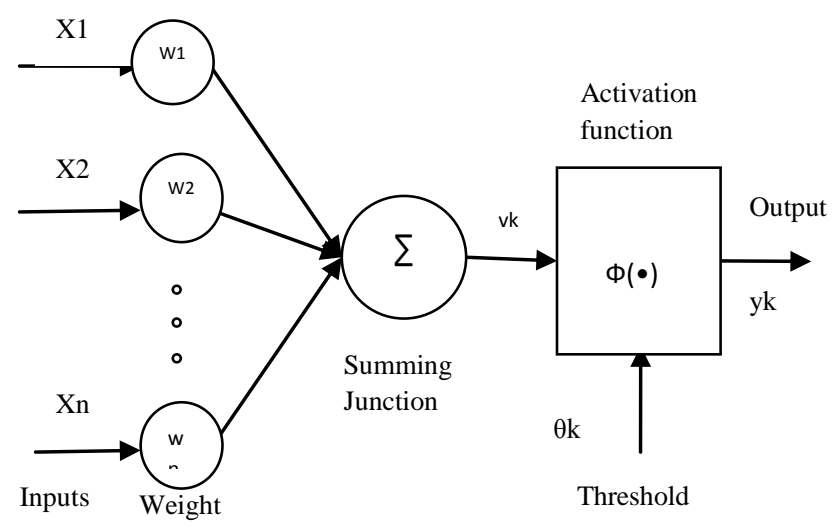

Figure 3. Mathematical model of neuron

$\mathbb{V}_{k}=\sum_{j=1}^{W} W_{j} X_{j}$

Where,

$\mathbb{W}_{\mathrm{k}}$ is summation value

$W_{j}$ is weight between neurons,

$x_{0}$ is input values 
$Y_{k}=\varphi\left(v_{k}+\theta_{k}\right)$

Where,

$Y_{h}$ is target value,

$\mathbb{V}_{\mathrm{k}}$ is summation output,

$\theta_{\mathrm{k}}$ is Threshold value

One of the big challenges in ANN model is proper selection of the number of hidden layers and the number of hidden neurons present in the hidden layer. If it is not in proper number, then it will affect the learning capability and leads to complexity.

\section{PROPOSED MODEL}

The proposed Neuro-fuzzy model made up of several steps Fig. 4:

1) Construct back propagation networks (BPNs).

2) Generate fuzzy set with a triangular shaped membership function.

3) Aggregate fuzzy forecasts with BPN

4) Defuzzify the fuzzy output to crisp value using Radial Basis Function (RBF).

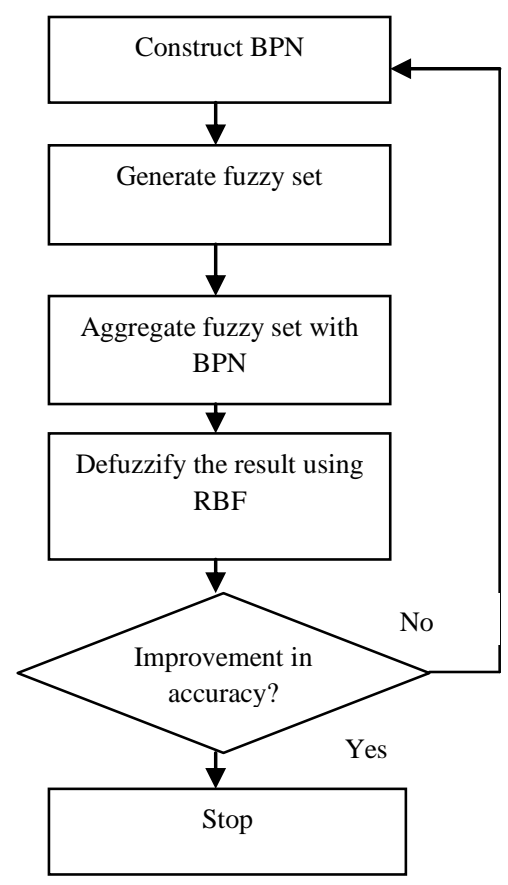

Figure. 4. Flowchart of neuro-fuzzy model

Step 1. Forecasting power consumption with BPNs:

i) Normalizing inputs - Population and GDP are the 2 input parameters, whose values should be normalized.

ii) Choosing of hidden layers -3 hidden layers are chosen as, one for fuzzification layer, the other for fuzzy rule layer and the third for output membership function.

iii) Selecting neurons - selection of neurons is based on the membership function and the fuzzy rule.

iv) Output - the annual power consumption.

v) Activation function - in this proposed model, sigmoid activation function is used.

vi) Epochs: 5000.
Step 2. Generate fuzzy set with a triangular-shaped membership function.

In this step, the annual power consumption value is generated, by using the triangular shaped membership function. This is mainly done to improve the accuracy of the power consumption value. Based on the membership function values as low, medium, high, the fuzzy rules are constructed.

Step 3. Defuzzify the aggregation result with Radial Basis Function (RBF).

In this step, defuzzufication is done by using the transfer function (RBF) and it is also called as sigmoid function. Defuzzification is the process of converting fuzzy value into crisp value. Among the several transfer functions, Gaussian transfer function is used.

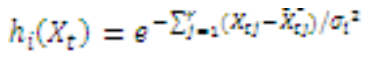

Where $h_{1}\left(X_{t}\right)$ is the output of defuzzification layer,

$$
\begin{array}{ll}
Z_{\mathrm{t}} & \text { is incoming vectors, } \\
Z_{\mathrm{t} j} & \text { is the center of the RBF, } \\
\sigma_{\mathrm{d}} & \text { is the width of the RBF. }
\end{array}
$$

The output layer:

$$
\begin{aligned}
& o\left(X_{t}\right)=\sum_{i=1}^{\mathbb{D}} w_{i} h_{i}\left(X_{t}\right)+W_{0} \\
& \text { where } o\left(X_{t}\right) \text { is the output value, } \\
& w_{i} \quad \text { is the weight, } \\
& h_{i}\left(X_{t}\right) \text { is the hidden layer output. }
\end{aligned}
$$

In order to determine the centre the clustering algorithm like k-means is used. To determine its width nearest neighbour method is used.
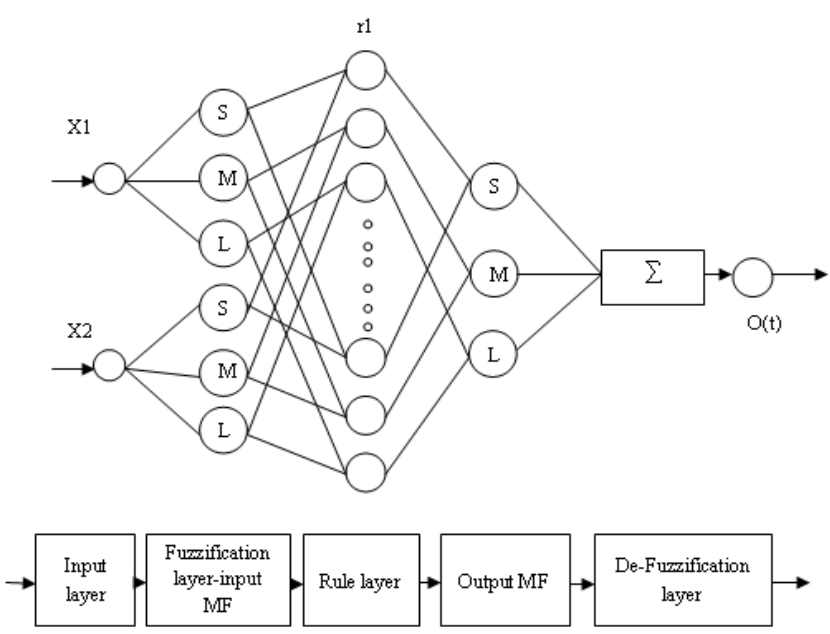

Figure 5. Neuro-fuzzy model

Figure. 5. Shows the neuro-fuzzy model for each layer.

Input Layer: The input layer neurons, which pass the input directly to the next hidden layer.

Fuzzification Layer: Each neuron in this layer represents a fuzzy set. In this proposed model triangular membership function is used. Each input is expressed in terms of membership values like Low, Medium and High. 
Fuzzy rule layer: In this layer, each neuron represents a fuzzy rule is formed [11]. These rules are formed by using the inputs obtained from the fuzzification layer. Based on the input and output fuzzy rules are formed. Triangular membership functions as shown in Fig. 6.

Output membership function: The output fuzzy set is constructed by using the fuzzy rules obtained from rule layer.

De-Fuzzification layer: This is the process of obtaining single output. In this model RBF de-Fuzzification method is used to defuzzify the values which are obtained from layer 4 .

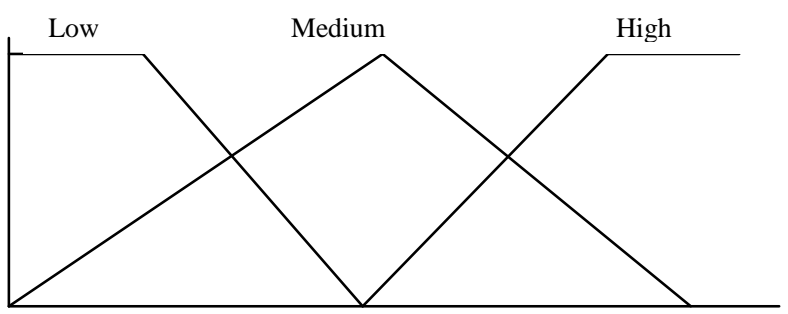

Figure 6. Triangular membership function

Table 1

ANN Power Consumption Output Based on one Hidden Layer with different Nodes

\begin{tabular}{|c|c|c|c|c|c|c|c|c|}
\hline \multirow{2}{*}{\multicolumn{2}{|c|}{ Actual output }} & \multicolumn{7}{|c|}{ Forecasted values } \\
\hline & & \multicolumn{7}{|c|}{ Number of hidden neurons } \\
\hline Year & $\begin{array}{l}\text { Units in } \\
\text { BkWh }\end{array}$ & 2 & 3 & 4 & 5 & 6 & 7 & 8 \\
\hline 1986 & 123.09 & 145.34 & 121.13 & 122.2 & 302.5 & 121.88 & 122.09 & 123.26 \\
\hline 2001 & 316.60 & 207.28 & 320.81 & 318.6 & 409.5 & 318.29 & 314.35 & 314.98 \\
\hline 2006 & 415.29 & 235.39 & 408.30 & 417.1 & 483.2 & 414.43 & 415.23 & 415.82 \\
\hline 2010 & 612.64 & 263.37 & 611.75 & 613.5 & 612.5 & 613.57 & 613.63 & 613.59 \\
\hline
\end{tabular}

\section{EXPERIMENTAL RESULT}

10 year historical data of power consumption, Population and GDP has been used for prediction. The historical population data was taken from population statistics webpage [13], GDP data was taken from indexmundi webpage [14] and the power consumption data was taken from iasri webpage [15]. Table 1 shows the existing ANN model forecasted values between 2 to 8 neurons for the year 1986, 2000, 2006, and 2010. In the existing ANN model the hidden layer neurons varied from 2 to 8 and found that the hidden layer with 8 neurons gives the best result among other neurons. Then neuro-fuzzy model is constructed and implemented using the same input GDP and Population which is used in ANN model. To evaluate the performance of proposed neuro-fuzzy model results are compared with neural networks with back propagation algorithm. Mean Absolute Percentage Error (MAPE) is used for the performance metrics.

$$
\text { MAPE }=\frac{\text { Actual waiwe-joxacagted walwe }}{20} \times 100
$$

Table 2 Power Consumption: Actual Vs Forecasted

\begin{tabular}{|c|c|l|c|c|c|}
\hline \multirow{2}{*}{ Year } & \multirow{2}{*}{$\begin{array}{c}\text { Actual } \\
\text { output } \\
(\text { BkW } \\
\text { h) }\end{array}$} & \multicolumn{2}{|c|}{$\begin{array}{c}\text { Forecasted Output } \\
\text { (BkWh) }\end{array}$} & \multicolumn{2}{c|}{ \%error } \\
\cline { 3 - 6 } & 123.09 & 123.26 & 122.58 & 0.14 & 0.41 \\
\hline 1986 & 126 & NF & ANN & NF \\
\hline 2001 & 316.60 & 314.98 & 316.54 & 0.51 & 0.018 \\
\hline 2006 & 415.29 & 415.82 & 415.36 & 0.13 & 0.016 \\
\hline 2010 & 612.64 & 613.59 & 612.35 & 0.16 & 0.047 \\
\hline \multicolumn{3}{|c|}{ Average absolute error } & 0.94 & 0.49 \\
\hline
\end{tabular}

Table 2 shows power consumption of actual and forecasted value using artificial neural network and neuro-fuzzy model for the year 1986, 2001, 2006 and 2010 and also it shows the error rate of actual and forecasted value. Table 3 shows power consumption of future years upto 2016 using ANN and NF model
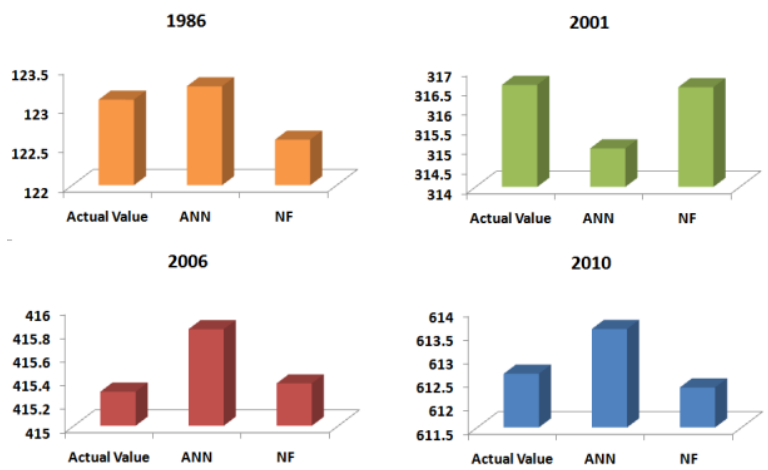

Figure 7. Power Consumption: Actual Vs Forecasted

Table 3 Forecasted Future Power Consumption: ANN and NF model

\begin{tabular}{|c|c|c|}
\hline \multirow{2}{*}{ Year } & \multicolumn{3}{|c|}{ Forecasted Output (BkWh) } \\
\cline { 2 - 3 } & ANN & NF \\
\hline 2013 & 716.4 & 912.4 \\
\hline 2014 & 758.38 & 979.2 \\
\hline 2015 & 802.52 & 1048.0 \\
\hline 2016 & 848.0 & 1118.5 \\
\hline
\end{tabular}




\section{CONCLUSION}

The power consumption of India is forecasted by using the social factors: GDP and Population. The two model Neurofuzzy and the ANN model of multilayer perceptron (MLP) with backpropagation network of different layer with different node have been studied and implemented using 30 years historical data and it has been tested with 4 years historical data. As the result shows that ANN model with one hidden layer with 8 neurons gives accurate result than the other neuron. The proposed neuro-fuzzy model is implemented using the same historical data which is used in the ANN model. The result of both models is validated using the MAPE. The MAPE obtained by ANN model is 0.94 and the proposed neuro-fuzzy Model is 0.49 . To conclude based on obtained result that the consumption using the neuro-fuzzy model is efficient and more accurate than the ANN model.

\section{REFERENCES}

[1]. S.Saravanan, S.Kannan, and C. Thangaraj, "Forecasting India's Electricity Demand Using Artificial Neural Network “, IEEE International conference 2012.

[2]. K. Hornik, M. Stinchcombe, and H. White, "Multilayer feed forward networks are universal approximators," in the proceeding of the IEEE Transaction on Neural Networks, vol. 2, no. 5, pp. 359-366, Oct 1995.

[3]. K. Hornik, "Approximation capabilities of multilayer feed forward networks," in the proceeding of the IEEE Transaction on Neural Networks, vol. 4, no. 2, pp. 251257, Jul 1991.

[4]. M. Leshno, V. Y. Lin, A. Pinkus, and S. Schocken, "Multilayer feed forward networks with a no polynomial activation function can approximate any function," in the proceeding of the IEEE Transaction on Neural Networks, vol. 6, no. 6, pp. 861-867, Aug 1993.

[5]. Manish Sankar, B. Yeganarayana, Deepak Khemani "Backpropagation learning algorithms for classification with fuzzy mean square error" Elsevier 1998.
[6]. RustumMamlook,OmarBadran ,EmadAbdulhadi, "A fuzzy inference model for short-term load forecasting " a Middle East University for Graduate Studies, Elsevier 2009.

[7]. D K Ranaweera, N F Hubele and G G Karady, "Fuzzy logic for short term load forecasting" in Elsevier transacting on Electrical \& Energy Systems Vol.18 No, pp.215-222,1996.

[8]. A. Ghanhari, A. Naghavi, S.F. Ghaderi, and M. Sabaghian, "Artificial Neural Networks and regression approaches comparison for forecasting Iran's annual electricity load," Proceeding International Conference on Power.

[9]. Bahman Kermanshahi, Hiroshi Iwamiya, Enviroinmental Engineering," Up to year 2020 load forecasting using neural nets", Elsevier 2002.

[10]. Paras Mandal, Tomonobu Senjyu, Naomitsu Urasaki, Toshihisa Funabashi, "A neural network based severalhour-ahead electric load forecasting using similar days approach" Elsevier 2006.

[11]. chih-chou chiu, deborah f. cook, jen-lung kao and yuchao chou " Combining a neural network and a rulebased expert system for short-term load forecasting " Elsevier 1997.

[12]. Stephan Trenn "Multilayer Perceptrons: Approximation Order and Necessary Number of Hidden Units" in the proceeding of the IEEE Transaction on Neural Networks, Vol.19. No.5, MAY 2008.

[13]. http://www.populstat.info/

[14]. http://www.indexmundi.com/india/gdp_(purchasing_po wer_parity).html

[15]. http://www.iasri.res.in/agridata/12data/chapter1/db2012 tb1_13.pdf 